\title{
A systematic review protocol on the epidemiology of zinc deficiency and associated factors during pregnancy in Africa
}

\author{
Melese L Endalifer ${ }^{1}$, Getnet G Azeze ${ }^{1}$, Gedefaw Diress ${ }^{2}$, Asmamaw D Bizuneh ${ }^{3}$, Hanna Demelash ${ }^{4}$ \\ 1 Department of Midwifery, Faculty of Health Science, Woldia University, Woldia, Ethioipia, ${ }^{2}$ Department of Public Health, Faculty of Health Science, \\ Woldia University, Woldia, Ethioipia, ${ }^{3}$ Department of Nursing, Faculty of Health Science, Woldia University, Woldia , Ethioipia, 4 Department of \\ Nutrition, College of Health and Medicine, Bahir Dar University, Bahir Dar, Ethioipia \\ Keywords: africa, maternal health, pregnancy, zinc deficiency \\ https://doi.org/10.29392/001c.12501
}

\section{Journal of Global Health Reports}

Vol. 4, 2020

\section{Background}

Zinc is a trace element which acts as a coenzyme for 200 enzymes. Zinc deficiency has highly correlated with infant morbidity and mortality. Even though zinc deficiency is an emergent public health problem, determinants and magnitude is not well studied in Africa. This study will estimate the pooled prevalence of zinc deficiency in Africa and to identify associated factors for zinc deficiency among pregnant women.

\begin{abstract}
Methods and analysis
We will use the following databases: PubMed, Google Scholar, and Cochrane library. Funnel plot and Egger's regression test will be conducted to check potential publication bias. Heterogeneity among the studies will be tested using Higgins method where I2 statistics will be calculated and compared with the standard. Meta regression and sub group analysis will be conducted to identify potential source of heterogeneity. We will include observational studies including case-control, cohort, and cross-sectional studies. The current review will delimit to studies conducted in Africa and published in English language.
\end{abstract}

\section{Ethics and dissemination}

Ethics approval and consent are not required. After completing the review, the paper will be submitted to a reputable peer reviewed journal.

PROSPERO registration number: CRD42019119449.

Micronutrient deficiency is one of the commonest public health problems in Africa. ${ }^{1}$ Primarily the principal causes are due to inadequate intake of zinc rich source food and absorption impairment in the intestine. ${ }^{2}$ Micronutrient deficiency especially zinc deficiency has major effect on the development of the fetus and the mother. ${ }^{1}$ Adequate maternal nutrition, particularly in prenatal period and during pregnancy is crucial for the health of the mother and their offspring. Poor nutrition in pregnancy period may lead to nutrient imbalance for the mother and fetus, which leads to adverse outcome. ${ }^{3-5}$ Adequate amount of $\mathrm{Zn}$ is necessary for normal pregnancy outcome and child growth, immune function and neurobehavioral development. ${ }^{6}$

Zinc deficiency during pregnancy and lactation leads to low birth weight, intrauterine growth restriction, impaired cognitive function, decreased production of milk, pregnancy induced hypertension, premature rupture of membrane, placental abruption, prolonged labor, hemorrhage, infections, congenital anomalies, increased neonatal morbidity and mortality and poor neurobehavioral development. ${ }^{1,7}$

Women who are living in tropical regions are suffered from infections disease like endemic malaria. Practicing zinc supplementation program for pregnant women in- creased acquired immunity and also important to prevent infectious disease. ${ }^{8}$ Pregnant women from subsistence farming households in rural Africa usually consume predominantly plant-based diets; intakes of animal-source foods were inadequate .As a result, micronutrient intakes are often inadequate. ${ }^{9,10}$

Zinc supplementation is not only affects the fetal growth and development but it has also significant effect on the other micronutrients bioavailability in the body particularly iron and iodine. ${ }^{11}$

Zinc deficiency is categorized as severe, moderate and mild. Despite, severe zinc deficiency is rare, mild and moderate zinc deficiency is increasing globally. ${ }^{12}$ In cases of severe $\mathrm{Zn}$ deficiency, skin lesions, anemia, diarrhea, anorexia, decreased lymphocyte function, impaired visual function and mental retardation may be observed. ${ }^{13}$

Globally, around $82 \%$ of pregnant women have experienced a lower zinc intake capacity as compared to the recommended dietary intake. Developed countries are trying to decrease the incidence of zinc deficiency via supplementation, but low and middle income countries are unable to decrease the magnitude of zinc deficiency and this may nearly around $100 \% .^{14}$ 
Household food insecurity, inadequate dietary diversity, low consumption of animal source food, low socioeconomic status, low-level maternal education, increasing parity, low hemoglobin concentration, maternal workload, chronic medical illness, being complete vegetarian and absence of antenatal care follow up are the major contributing factors for zinc deficiency.

\section{RATIONALE FOR CURRENT SYSTEMATIC REVIEW}

The magnitude of zinc deficiency is increasing globally and shows increasing trend in Africa. Despite, different studies conducted in Africa, the magnitude of zinc deficiency and determinant factors were not studied yet. Therefore, we draft this protocol to estimate the magnitude of zinc deficiency among pregnant women in Africa. Estimating the prevalence of zinc deficiency during pregnancy is a direct indicator of fetal growth and development. Additionally providing comprehensive evidence is important for implementation of zinc supplementation, fortification, diversification and nutritional education program in the continent.

\section{METHODS}

\section{OBJECTIVES}

To conduct a systematic review and meta-analysis on the pooled prevalence of Zinc deficiency and its associated factors among pregnant women.

\section{REVIEW QUESTIONS}

- What is the prevalence zinc deficiency in African among pregnant women?

- What are the factors that associated with zinc deficiency among pregnant women in African?

\section{ELIGIBILITY CRITERIA}

\section{INCLUSION CRITERIA}

1. Pregnant women in Africa.

2. Papers published only in English Language from 2010 to 2018.

3. This review will includes all observational studies: cross sectional, case control, trend, prospective and retrospective cohort studies.

\section{EXCLUSION CRITERIA}

1. Pregnant women with special health problems like HIV/AIDS, chronic health problem and exclusively at higher risk of zinc deficiency

2. Editorials, reports, meta-analyses, systematic review, case reports, proceedings, grey literatures and case series

3. Studies those participants who are not human.

\section{DATABASES AND SEARCHING STRATEGY}

1. We will search articles from the following data bases which are directly related to the subject matter under review. Cochrane library; Pub Med, Google scholar and retrieving references from a list of eligible studies will be employed. Additionally articles from American Journal of Clinical Nutrition and Nutrition Journal email alerts will be searched.

2. We will supplement our database searches through manually searching the reference lists of all included papers and relevant reviews.

3. We will apply Boolean operator that is "AND", "NOT" and "OR". Through consideration of the Boolean operator we searched as follows((zinc OR zinc[MeSH Terms] AND ( pregnancy* OR pregnant OR pregnant women OR pregnant mother OR Women OR mothers) AND (Africa OR African Countries OR East Africa OR West Africa OR North Africa OR South Africa OR Central Africa OR Sub Saharan Africa)).

\section{DATA EXTRACTION AND MANAGEMENT}

Standard systematic searching will be conducted from those databases. Studies conducted before 2010, conducted other than African countries and unrelated articles based on their title will be excluded. Those potentially eligible studies will be imported to Endnote version 7 and exact duplicates will be removed. Two independent reviewers will done abstract and full-text review and data abstraction. In case of disagreement between the two reviewers, discussion will be made to reach consensus. Lastly, the studies will be screened and selected for full-text review based on the following inclusion criteria. The PRISMA statement for reporting systematic review and meta-analysis will be used to present the study inclusion, exclusion and reason for exclusion information in diagram.

\section{APPRAISAL OF THE QUALITY OF INCLUDED STUDIES}

Two reviewers will examine the quality of the included studies. Newcastle-Ottawa Scale (NOS) will be used to assess the quality of included studies. The NOS includes 3 categorical criteria with a maximum score of 9 points; "selection" which accounts a maximum of 4 points, "comparability" which accounts a maximum of 2 points, and "outcome" which accounts a maximum of 3 points. The quality of each study will be rated using the following scoring algorithm: $\geqslant 7$ points will be considered as "good", 2 to 6 points will be considered as "fair", and $\leqslant 1$ point will be considered as "poor" quality study. The scoring of each quality assessment component for each study will be presented in table. In addition to quality assessment, the reporting of the systematic review and meta-analysis result will be based on Meta-analysis Of Observational Studies in Epidemiology (MOOSE) statement.

\section{DATA SYNTHESIS INCLUDING ASSESSMENT OF HETEROGENEITY}

Prevalence of zinc deficiency among pregnant women from each study will be pooled and determine as a single estimate. Funnel plot and Egger's regression test will be conducted to check potential publication bias. In case of minor publication bias, Trim and Fill analysis will be used as ad- 
justment. Heterogeneity among the studies will be tested using Higgins et al method where $I^{2}$ statistics is calculated and compared with the standard. Meta regression analysis will be conducted to identify potential source of heterogeneity in the studies.

Risk factors obtained from each primary studies will be thematically organized and their effect size will be Metaanalyzed accordingly. If there is a minor heterogeneity, a separate forest plot will be constructed based on the identified source of heterogeneity, if not, narrative synthesis will be conducted. The data will be imported and analyzed using the STATA version 14 software (StataCorp, College Station, TX, USA).

\section{SUB-GROUP ANALYSIS}

Sub-group analysis will be conducted based on type of zinc deficiency measurement, geographical location and based on meta-regression output.

\section{PRESENTING AND REPORTING THE RESULTS}

We will use a PRISMA flow chart to summarize the study selection process and to show the excluded articles and rationale for exclusion. The prevalence of zinc deficiency will be presented in a forest plot or individually in tables.

\section{STRENGTHS AND LIMITATIONS OF THE STUDY}

- The strength of this study is including wide geographical areas.

- The drafted systematic review and meta-analysis review will provide an overall prevalence of zinc deficiency in Africa.

- The proposed systematic review and meta-analysis will stick to the Preferred Reporting Items for Systematic Reviews and Meta Analyses (PRISMA) guidelines, ensuring reliability in reporting and the full systematic review.

- The proposed systematic review and meta-analysis will offer the magnitude of zinc deficiency among pregnant women.
- The limitation this study is that it doesn't include literatures published other than English language.

\section{CONCLUSIONS}

These systematic review and Meta analyses data will be extracted using a structured data extraction form and presented using tables. The name of author, year of publication, country,residence, study setting, study design, sample size, tool used to screen undernutrition, cut of value, prevalence, associated factors with odds ratio and 95\% conficence intervals were summarized. Before analysis, transforamtion of odds ratios and prevalences will be made.

Ethics: Not applicable.

Funding: None.

Author's contributions: MLE, GGA, GD, ADB and HD participated in study protocol development, data collection, analysis and write-up. All authors read and approved the final manuscript.

Competing interests: The authors completed the Unified Competing Interest form at www.icmje.org/coi_disclosure.pdf (available upon request from the corresponding author), and declare no conflicts of interest.

\section{Correspondence to:}

Melese Linger Endalifer

Department of Midwifery

Faculty of Health Science

Woldia University

Woldia

Ethioipia

melselinger@gmail.com 


\section{REFERENCES}

1. Ejezie FE, Nwagha UI. Zinc Concentration during Pregnancy and Lactation in Enugu, South-East Nigeria. Ann Med Health Sci Res. 2011;1:69-76.

2. Tuerk MJ, Fazel N. Zinc deficiency. Curr Opin Gastroenterol. 2009;25:136-143. doi:10.1097/MOG.0b 013e328321b395

3. Black RE, Victora CG, Walker SP, et al. Maternal and child undernutrition and overweight in low-income and middle-income countries. Lancet. 2013;382:427-451. doi:10.1016/S0140-6736(13)6093 7-X

4. Grieger JA, Clifton VL. A review of the impact of dietary intakes in human pregnancy on infant birth weight. Nutrients. 2015;7:153-178.

5. Gluckman PD, Hanson MA. Developmental origins of disease paradigm: A mechanistic and evolutionary perspective. Pediatr Res. 2004;56:311-317.

6. Skeaff SA. Iodine deficiency in pregnancy: The effect on neurodevelopment in the child. Nutrients. 2011;3:265-273. doi:10.3390/nu3020265

7. Gebremedhin S, Enquselassie F, Umeta M. Prevalence of prenatal zinc deficiency and its association with socio-demographic, dietary and health care related factors in Rural Sidama, Southern Ethiopia: A cross-sectional study. BMC Public Health. 2011;11:898. doi:10.1186/1471-2458-11-898

8. Saaka M, Oosthuizen J, Beatty S. Effect of prenatal zinc supplementation on birth weight. J Health Popul Nutr. 2009;27:619-631. doi:10.3329/jhpn.v27i5.3638
9. Gebre-Medhin M, Gobezie A. Dietary intake in the third trimester of pregnancy and birth weight of offspring among nonprivileged and priviledged women. Am J Clin Nutr. 1975;28:1322-1329. doi:10.1 093/ajen/28.11.1322

10. Huddle J-M, Gibson RS, Cullinan TR. The impact of malarial infection and diet on the anemia status of rural pregnant Malawian women. Eur J Clin Nutr. 1999;53:792-801. doi:10.1038/sj.ejen.1600851

11. Siyame EW, Hurst R, Wawer AA, Young SD, Broadley MR, Chilimba AD, et al. A high prevalence of zinc- but not iron-deficiency among women in rural Malawi: A cross-sectional study. Int J Vitam Nutr Res. 2013;83:176-187.

12. Chiplonkar SA, Kawade R. Effect of zinc- and micronutrient rich food supplements on zinc and vitamin A status of adolescent girls. Nutrition. 2012;28:551-558. doi:10.1016/j.nut.2011.08.019

13. McClain CJ, McClain M, Barve S, Boosalis MG. Trace metals and the elderly. Clin Geriatr Med. 2002;18:801-818. doi:10.1016/S0749-0690(02)0004 $\underline{0-X}$

14. Caulfield LE, Zavaleta N, Shankar AH, Merialdi M. Potential contribution of maternal zinc supplementation during pregnancy to maternal and child survival. The American Journal of Clinical Nutrition. 1998;68(2):499S-508S. doi:10.1093/ajcn/6 $\underline{8.2 .499 \mathrm{~s}}$ 